\title{
Effect of a moving boundary on the deformation of a poro-elastic cylinder
}

\author{
S. I. Barry*and G. N. Mercer ${ }^{\dagger}$ \\ (Received 28 August 1996) \\ (Revised 25 November 1997)
}

\begin{abstract}
The deformation of a poro-elastic cylinder due to radial fluid flow is considered. This has application to modelling arterial flow and certain filtration processes. A diffusion equation for the dilatation with unusual integral boundary conditions is derived for two typical boundary conditions. Asymptotic solutions, to the linearised equations for small times, are found using
\end{abstract}

* School of Mathematics and Statistics, University College, University of New South Wales, Canberra, A.C.T 2600, Australia. mailto:s-barry@adfa.oz . au

${ }^{\dagger}$ School of Mathematics and Statistics, University College, University of New South Wales, Canberra, A.C.T 2600, Australia. mailto:g-mercereadfa.oz . au

See http:// jamsb.sci.usq.edu.au/V39/E004/ for this paper and ancillary services, (c) Austral. Mathematical Soc. 1998. Published 2 Feb 1998, last updated February 3, 1998. 
Maple and shown to be remarkably accurate even for relatively large times. Since the position of the boundaries changes with time, the fully nonlinear system is solved numerically as a moving boundary value problem. Solutions for the dilatation and displacement are found and comparisons made between the standard linearised and full moving boundary problems with a nonlinear, strain dependent permeability. It is shown that inclusion of the correct position of the moving boundaries has a comparable effect to inclusion of a nonlinear permeability on the deformation of the cylinder. 


\section{Contents}

1 Introduction $\quad 630$

2 Poro-Elastic Theory $\quad 633$

$3 \quad$ Developing the Governing Equations $\quad 637$

4 Boundary Conditions

5 Non-Dimensionalisation $\quad 641$

6 Numerical Solution $\quad 643$

$7 \quad$ Small Time Solution $\quad 643$

$\begin{array}{llr}8 & \text { Steady State Solution } & 647\end{array}$

9 Results and Discussion $\quad 647$

10 Conclusion $\quad \mathbf{6 5 6}$

$\begin{array}{lr}\text { A Appendix } & 657\end{array}$

$\begin{array}{lr}\text { References } & 663\end{array}$ 


\section{Introduction}

Poro-elasticity describes the coupled interaction between fluid flow and solid deformation in saturated porous materials. The pressure gradient, driving fluid through a porous solid, deforms the solid which in turn may affect the passage of fluid. Poro-elastic modelling is common in both engineering and biology, in particular the modelling of soils and soft biological tissue.

Arteries are an example of a biological tissue which may be modelled by poroelasticity $[14,15,16,17,11,10]$. The small transmural fluid flux through the artery wall is coupled to the deformation of the artery. If the porosity of the artery changes, due to the applied fluid pressure, then this may enhance or restrict the flux of fluid and proteins through the wall. This process may be linked to atherogenesis - an accumulation of proteins within the artery wall [24, 25, 20]. An understanding of the internal deformation of the artery is hence a necessary link in understanding the relationship between fluid flux, protein accumulation and applied fluid pressure. Arteries have a complicated structure which homogeneous poro-elastic models can not model. However, such models can lead to greater understanding of the interaction between flow and deformation within the artery, particularly when compared to in vitro studies of arterial transport such as [20].

Mathematical models of poro-elasticity began with studies of soil consolidation $[27,5]$ which have been used extensively in soil science. Kenyon [13] used the theory of mixtures to derive similar equations governing poro-elasticity which he used to model arterial transport $[14,15]$. The poro-elastic equations were fur- 
ther developed (for example $[19,22,23]$ ) to model the deformation and lubrication properties of articular cartilage, including the effect of strain dependent permeability [19], nonlinear stress-strain relations [23] and visco-elastic solid components [21].

There have been several models of fluid flux through poro-elastic cylinders or models of transport in arteries. Kenyon $[14,15]$ looked at the steady state and small time response (due to a step change in pressure) of arteries using linear poroelasticity. Jayaraman [11] and Jain and Jayaraman [10] considered an oscillatory pressure gradient using linear poro-elasticity. The effect on arterial transport of a nonlinear dependence of permeability on strain was studied by Klanchar and Tarbell [16] who looked at the steady state relationship between applied pressure and velocity flux. This work was extended by Kim and Tarbell [17] to consider macromolecular transport within the arterial wall. The mechanics of thin poroelastic shells has also been studied [26]. In most of these publications the governing equation (13) was used to find the displacement. An alternative approach was used by Barry and Aldis [4] who solved a linear equation for the dilatation, independent of the velocity or displacement. They found analytical solutions for radial flow through a porous cylinder with an outer boundary constrained by a rigid, impermeable mesh.

Nonlinearities appear in poro-elasticity due to a variety of causes. The dependence of permeability on strain (for example $[19,7,8,2]$ ) and nonlinear stressstrain relations $[23,9,3]$ are often considered in poro-elastic modelling. The position of the boundaries, however, also cause nonlinearities to occur although few authors have studied the magnitude of this effect in poro-elasticity. Jensen et al. 
[12] incorporated the moving boundary position when they considered the relaxation of a porous deformable layer. They derived a nonlinear diffusion equation for the surface height with a perturbation correction for the boundary position.

In this study we extend the work of Barry and Aldis [4] to consider the effect of the moving boundaries involved in the deformation of poro-elastic cylinders. In infinitesimal, linear poro-elasticity, the equations are represented in an Eulerian (spatial) reference frame but the boundary conditions are applied at the original position of the boundary. This assumes that the deformation is small so that the final and original positions of the boundaries are sufficiently similar and hence the resulting errors are minimal. With poro-elastic materials, however, the deformations are often much larger than found in other elastic materials (such as steel or rock) thus violating the infinitesimal assumption. Two standard methods are often used to compensate for the boundary position. First, the equations can be converted to a Lagrangian (material) reference frame. Second, the equations can be posed in Eulerian coordinates and solved as a moving boundary value problemthat is, where the position of the boundary is an unknown to be determined from the analysis. Barry and Aldis [3] considered the Lagrangian approach in solving a one-dimensional steady deformation with various strain dependent permeabilities. It will be shown, however, that conversion to a Lagrangian framework is difficult for the time dependent deformation considered here. We thus solve the governing equations as a moving boundary problem and compare our solution with the fully linear system. The effect of a strain dependent permeability is also considered.

We make several standard assumptions. The elastic solid matrix is initially homogeneous and isotropic and there exist no external body or osmotic forces 
acting on the medium. The individual components of the mixture are intrinsically incompressible. Bulk compression arises from a reduction in the fluid volume fraction rather than compression of the individual constituents. Inertia is also assumed negligible [2] on the time scales appropriate to this problem. We consider only a linear dependence of stress on strain.

An axisymmetric cylindrical geometry is used as illustrated in Figure 1. Fluid flow is radially outwards through the medium driven by a pressure $p=p_{0}>0$ on the inner surface and $p=0$ on the outer surface. The inner surface is assumed to have zero solid contact stress. Two conditions are considered for the outer boundary. These represent the two possible extremes. Figure 1(a) illustrates a cylinder constrained by a rigid, permeable mesh which limits the expansion of the cylinder. In Figure 1(b) the cylinder is free to expand as the outer boundary is assumed to have zero contact stress. The inner radius is initially at $r=\alpha$ and the outer boundary is initially at $r=\beta$.

In the next section we develop the poro-elastic model. The governing equations and boundary conditions will then be derived. A description of the numerical scheme used to solve the moving boundary problem is then given, followed by an analytical solution for linear poro-elasticity. Results for the deformation and dilatation are then described and discussed.

\section{Poro-Elastic Theory}


(a) constrained cylinder

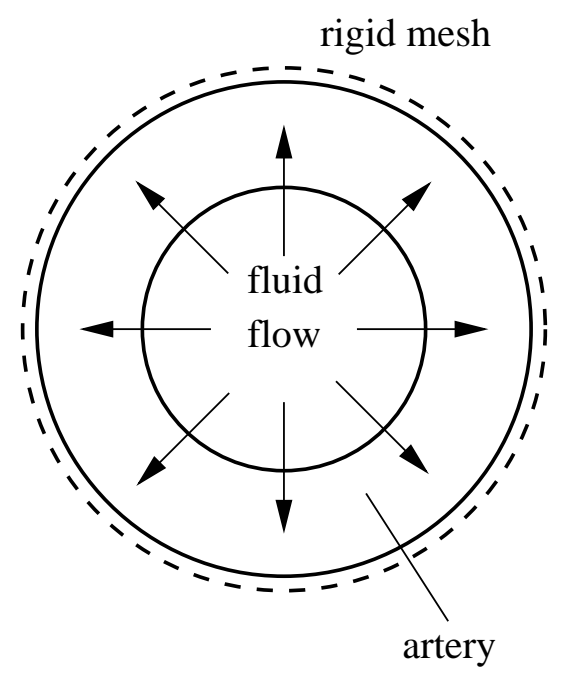

(b) unconstrained cylinder

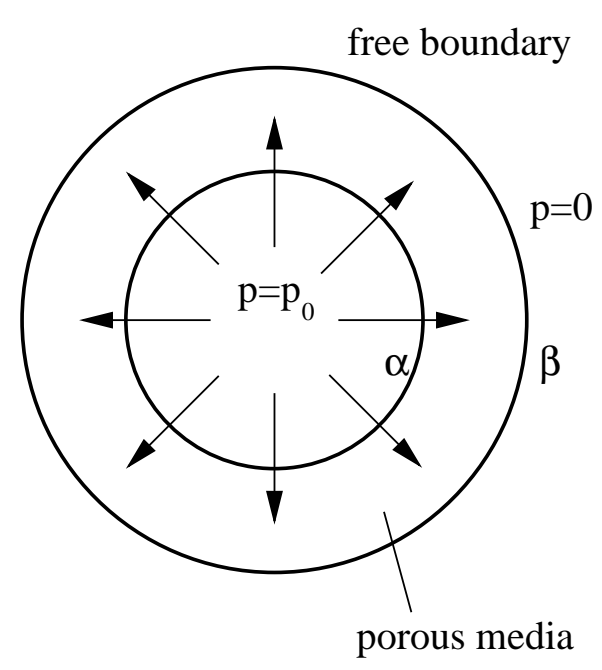

cross section

FIGURE 1. Schematic diagram of a cylinder with inner radius $\alpha$ and outer radius $\beta$ deforming due to an applied pressure difference $p=p_{0}$. (a) The cylinder is constrained by rigid, permeable outer mesh. (b) The outer boundary of the cylinder can expand and has zero contact stress. 
The aim of this section is to briefly derive the equations governing poro-elasticity using mixture theory (see for example [7, 8, 23, 4]).

The volume occupied by the $\gamma=s, f$ (solid,fluid) phase is denoted $V^{\gamma}$ with mass $m^{\gamma}$. The apparent density $\rho^{\gamma}$, intrinsic density $\rho_{T}^{\gamma}$, and volume fraction $\phi^{\gamma}$ are defined as

$$
\rho^{\gamma}=\lim _{d V \rightarrow 0} \frac{d m^{\gamma}}{d V}, \quad \rho_{T}^{\gamma}=\lim _{d V^{\gamma} \rightarrow 0} \frac{d m^{\gamma}}{d V^{\gamma}}, \quad \phi^{\gamma}=\lim _{d V \rightarrow 0} \frac{d V^{\gamma}}{d V} .
$$

The intrinsic density is a constant by the assumption of incompressibility while the apparent density and volume fraction may change as the proportion of solid and fluid change.

A continuity equation,

$$
\nabla \cdot \mathbf{v}=0
$$

can be derived [23] where $\mathbf{v}=\phi^{f} \mathbf{v}^{f}+\phi^{s} \mathbf{v}^{s}$ is a volume averaged velocity. The velocities of the fluid, $\mathbf{v}^{f}$, and solid, $\mathbf{v}^{s}$, represent velocities relative to an external reference frame.

The momentum equation for each phase is

$$
\nabla \cdot \mathbf{T}^{\gamma}=-\boldsymbol{\pi}^{\gamma},
$$

where $\boldsymbol{\pi}^{\gamma}$ is a drag force on the $\gamma=s, f$ phase and $\mathbf{T}^{\gamma}$ the total stress on each phase. Here we assume no external body forces and neglect inertial terms. 
The stress tensors are modelled as

$$
\begin{aligned}
& \mathbf{T}^{f}=-\phi^{f} p \mathbf{I}, \\
& \mathbf{T}^{s}=-\phi^{s} p \mathbf{I}+\boldsymbol{\sigma},
\end{aligned}
$$

where $\sigma$ represents a solid contact stress, a function of the strain, $p$ is the fluid pressure and $\mathbf{I}$ the identity tensor. Here we assume that there is no viscous shearing stress in the fluid.

Newton's third law implies $\boldsymbol{\pi}^{s}=-\boldsymbol{\pi}^{f}$ : the force on the solid by the fluid is opposite to the force on the fluid by the solid. The friction drag terms are modelled as

$$
-\boldsymbol{\pi}^{s}=\boldsymbol{\pi}^{f}=K\left(\mathbf{v}^{s}-\mathbf{v}^{f}\right)-p \nabla \phi^{s},
$$

where $K$ is the drag coefficient of relative motion. This is essentially Darcy's law.

The basic relationships between pressure, displacement and velocity are found by substituting (4) into (3) and adding both phase equations to eliminate $\pi^{\gamma}$. Substituting the interaction term (5) into (3) and making use of $\phi^{s}=1-\phi^{f}$ leads to

$$
\nabla p=\nabla \cdot \boldsymbol{\sigma}=\frac{1}{k}\left\{\frac{\partial \mathbf{u}}{\partial t}-\mathbf{v}\right\},
$$

where $k=\left(\phi^{f}\right)^{2} / K$ is the permeability [18], $\mathbf{u}$ is the displacement of the solid and $\partial \mathbf{u} / \partial t=\mathbf{v}^{s}$. 
Equation (6) is easily explained in physical terms. Darcy's law expressed relative to the movement of the solid is $\nabla p=1 / k(\partial \mathbf{u} / \partial t-\mathbf{v})$. If the stress in the solid matrix is governed by the standard equilibrium equation of elasticity then $\nabla \cdot \boldsymbol{\sigma}=\nabla p$ where the gradient of pressure is acting as an internal body force on the solid matrix.

As a porous medium is compressed the resulting decrease in porosity will lead to a reduction in the permeability $k$. The simplest model that allows for this is if $k=k(\phi)$ where $\phi=\nabla \cdot \mathbf{u}=\left(\phi^{f}-\phi_{0}^{f}\right) / \phi_{0}^{s}$ is the dilatation of the medium and $\phi_{0}^{\gamma}$ are the initial volume fractions of the solid and fluid phases. Various forms for this functional dependence have been considered $[8,3]$ and it seems that the most versatile is an exponential dependence given by $k=k_{0} e^{m \phi}$ where $k_{0}$ and $m$ are constants.

\section{Developing the Governing Equations}

The stress tensor is related to the strain tensor by the standard linear relationship

$$
\boldsymbol{\sigma}=\lambda e_{k k} \mathbf{I}+2 \mu \mathbf{e}, \quad \mathbf{e}=\frac{1}{2}\left(\nabla \mathbf{u}+(\nabla \mathbf{u})^{T}\right)
$$

where $\mathbf{e}$ is the strain tensor and $\lambda$ and $\mu$ are the Lamé stress constants.

Assuming a purely radial displacement $(u(r), 0,0)$ in cylindrical coordinates 
$(r, \theta, z)$, the only non zero stress components are:

$$
\sigma_{r r}=(\lambda+2 \mu) \frac{\partial u}{\partial r}+\lambda \frac{u}{r}, \quad \sigma_{\theta \theta}=(\lambda+2 \mu) \frac{u}{r}+\lambda \frac{\partial u}{\partial r},
$$

and

$$
(\nabla \cdot \boldsymbol{\sigma})_{r}=\frac{\partial \sigma_{r r}}{\partial r}+\frac{\sigma_{r r}-\sigma_{\theta \theta}}{r} .
$$

It can also be shown, with a little algebra, that substitution of (8) into (9) gives

$$
(\nabla \cdot \boldsymbol{\sigma})_{r}=H_{a} \frac{\partial \phi}{\partial r}
$$

where

$$
\phi=\frac{1}{r} \frac{\partial}{\partial r}(r u),
$$

is the dilatation and $H_{a}=\lambda+2 \mu$ is the aggregate elastic modulus.

Integration of the continuity equation (2) leads to the velocity component in the radial direction

$$
v_{r}=\frac{v(t)}{r},
$$

for some function $v(t)$. Equation (6) then becomes

$$
\frac{\partial p}{\partial r}=H_{a} \frac{\partial}{\partial r}\left\{\frac{1}{r} \frac{\partial}{\partial r}(r u)\right\}=\frac{1}{k(\phi)}\left\{\frac{\partial u}{\partial t}-\frac{v(t)}{r}\right\} .
$$

By applying the operator

$$
L[w]=\frac{1}{r} \frac{\partial}{\partial r}(r w)
$$


to both sides of (13) we obtain

$$
H_{a} \frac{1}{r} \frac{\partial}{\partial r}\left\{k(\phi) r \frac{\partial \phi}{\partial r}\right\}=\frac{\partial \phi}{\partial t}
$$

which is a diffusion equation independent of the velocity and the displacement.

It will be shown in the next section that the boundary conditions for equation (15) can also be expressed independent of the velocity. This is a more useful form of the governing equations than (13) since experimentally the internal pressure is often applied with the fluid velocity only inferred.

\section{Boundary Conditions}

We now consider the boundary conditions shown in Figure 1(b), the unconstrained cylinder. The linearised boundary conditions for the constrained cylindrical shell are discussed in Barry and Aldis [4].

Since both the inner and outer boundaries have no contact stress [6] then

$$
\sigma_{r r}(\alpha, t)=0, \quad \sigma_{r r}(\beta, t)=0 .
$$

This can be written as

$$
\left[\frac{\partial u}{\partial r}+\left(1-\lambda_{2}\right) \frac{u}{r}\right]_{r=\alpha, \beta}=0
$$


where $\lambda_{2}=2 \mu /(\lambda+2 \mu)$. In terms of $\phi$ this is

$$
\left[\phi(r, t)-\lambda_{2} \frac{u(r, t)}{r}\right]_{r=\alpha, \beta}=0 .
$$

To express this entirely in terms of $\phi$ we must find an expression for $u$ in term of $\phi$.

By integrating (11), the displacement can be written as

$$
u(r, t)=-\frac{1}{r} \int_{r}^{\beta} r \phi(r, t) d r+\frac{\phi(\beta, t) \beta^{2}}{\lambda_{2} r}
$$

where the boundary condition at $r=\beta$, equation (17), has been used.

Integrating the relation between pressure and stress, equation (13), the boundary condition at the constrained boundary, $r=\beta$, is

$$
\phi(\beta, t)=\phi(\alpha, t)-\frac{p_{0}(t)}{H_{a}} .
$$

Using (19) and (20) in (18) we obtain

$$
\phi(\alpha, t)=\frac{\lambda_{2}}{\beta^{2}-\alpha^{2}} \int_{\alpha}^{\beta} r \phi(r, t) d r+\frac{p_{0}}{H_{a}} \frac{\beta^{2}}{\beta^{2}-\alpha^{2}} .
$$

Hence (21) and (20) are the boundary conditions to be used in conjunction with equation (15). 


\section{Non-Dimensionalisation}

To simplify the equations we non-dimensionalise all variables using the relationships:

$$
p=H_{a} \hat{p}, \quad r=\beta_{0} \hat{r}, \quad k=k_{0} \hat{k}, \quad u=\beta_{0} \hat{u}, \quad \alpha=\beta_{0} a, \quad \beta=\beta_{0} b
$$

and

$$
t=\frac{\beta_{0}^{2}}{H_{a} k_{0}} \hat{t}
$$

where $\beta_{0}$ is the initial position of the outer boundary.

For ease of notation we will now drop the hat notation denoting non-dimensional quantities and all variables will now be considered non-dimensional. The only parameters in the system are the scaled initial inner radius, $a_{0}$, the elastic parameters, $\lambda_{2}=2 \mu /(\lambda+2 \mu)$, and the scaled applied pressure, $p_{0}$.

In non-dimensional form, the governing equations for this system become:

$$
\begin{aligned}
\frac{1}{r} \frac{\partial}{\partial r}\left\{k(\phi) r \frac{\partial \phi}{\partial r}\right\} & =\frac{\partial \phi}{\partial t} \\
\phi(a, t) & =\frac{\lambda_{2}}{b^{2}-a^{2}} \int_{a}^{b} \alpha \phi(\alpha, t) d \alpha+p_{0} \frac{b^{2}}{b^{2}-a^{2}} \\
\phi(b, t) & =\phi(a, t)-p_{0}(t)
\end{aligned}
$$

with inner radius $a(t)$ and outer radius $b(t)$. Initially

$$
\phi(r, 0)=0, \quad a(0)=a_{0}, \quad b(0)=1 .
$$


Since the distances have been scaled with respect to the initial position of the outer boundary, $b_{0}$, then $b(0)=1$.

The positions of the boundaries $a, b$ are unknowns. They are related to $\phi$ by the implicit relationships:

$$
a(t)=a_{0}+u(a(t), t), \quad b(t)=b_{0}+u(b(t), t),
$$

and

$$
u(r, t)=-\frac{1}{r} \int_{r}^{b} \alpha \phi(\alpha, t) d \alpha+\frac{\phi(b, t) b^{2}}{\lambda_{2} r}
$$

The equations (23-26) represent a nonlinear diffusion equation for $\phi$ with unusual integral boundary conditions. The unknown position of the boundaries are represented by equations (27) and (28). A full analytical solution is not possible for this nonlinear coupled system, however numerical solutions will be found. Analytical solutions for the linearised problem, where $a=a_{0}, b=1$ and $k=1$, will also be found using Laplace transforms.

The approach used in Barry and Aldis [3] — conversion to a Lagrangian (material) reference frame-will be ineffective for this set of equations. Relating the Lagrangian coordinate, $R$, to the Eulerian, $r$, by the displacement relationship, $r=U(R, t)+R$, implies that the Lagrangian displacement, $U$, would thus occur nonlinearly in equation (23). These equations would then be a mixture of variables $U$ and $\phi$ which would be difficult to solve. 


\section{Numerical Solution}

The numerical method employed to solve the equations (23-26) with the moving boundary conditions, equations (27-28), is an explicit finite difference Euler scheme with centred space derivatives. At each time step, the region $(a(t), b(t))$ is discretised into $N$ equal intervals. The value of $\phi$ at the internal mesh points is easily calculated from (23) for the next time step. By using Simpson's rule to calculate the nonlinear integral in the boundary condition, it is possible to determine the value of $\phi$ at the boundaries $(r=a, b)$ in terms of $\phi$ at the internal mesh points. Once these $\phi$ values have been determined over the entire domain, the displacements are determined using (28). The boundaries are then incremented and the procedure repeated.

This method is only first order accurate in time. Hence a small time step is required to ensure accuracy and stability of the solutions. Notwithstanding this, the method is fast, simple and agrees with the small time asymptotic results outlined in the next section for the case of constant permeability $k$. This numerical method can be used with any nonlinear permeability $k(\phi)$.

\section{Small Time Solution}

In this section we find solutions to equations (23-26) for an unconstrained cylinder. We assume a linearised problem where $k=1$ and, rather than equation (27), 
we set $a=a_{0}$ and $b=1$. The boundary conditions are hence applied at the original position of the boundaries with the conventional infinitesimal assumption. A Laplace transform technique will be used and an asymptotic solution for small times calculated. We will show, however, that this small time solution is valid almost up to the time when a steady state equilibrium is reached. This solution is also used to check the numerical scheme discussed in the previous section.

Taking the Laplace transform, $\mathcal{L}\{\phi(r, t)\}=\bar{\phi}(r, s)$, of (23) gives

$$
\eta^{2} \bar{\phi}^{\prime \prime}+n \eta \bar{\phi}^{\prime}-\eta^{2} \bar{\phi}=0
$$

where $\eta=r \sqrt{s}$. This has general solution

$$
\bar{\phi}(\eta, s)=A_{1}(s) I_{0}(\eta)+A_{2}(s) K_{0}(\eta),
$$

where $I_{0}, K_{0}$ are modified Bessel functions of order zero. The functions $A_{1}, A_{2}$ are found from application of the transformed boundary conditions:

$$
\begin{aligned}
\bar{\phi}(a, s) & =\frac{\lambda_{2}}{b^{2}-a^{2}} \int_{a}^{b} r \bar{\phi}(r, s) d r+\overline{p_{0}} \frac{b^{2}}{b^{2}-a^{2}}, \\
\bar{\phi}(b, s) & =\bar{\phi}(a, s)-\overline{p_{0}},
\end{aligned}
$$

where $\overline{p_{0}}=\mathcal{L}\left\{p_{0}\right\}$. We note that because of the infinitesimal assumption, $b=1$ in these equations. The resulting solution is clearer, however, if we do not make this substitution.

The applied pressure we use is $p_{0}=1$ for $t>0$ and $p_{0}=0$ for $t \leq 0$. This implies that $\overline{p_{0}}=1 / s$. More complicated functions for the applied pressure 
can be analysed but this simple form mimics many experimental procedures and illustrates the mechanics of the poro-elastic system clearly.

Substitution of (30) into the transformed boundary conditions (31-32) gives the system of equations:

$$
\left[\begin{array}{ll}
a_{11} & a_{12} \\
a_{21} & a_{22}
\end{array}\right]\left[\begin{array}{l}
A_{1} \\
A_{2}
\end{array}\right]=\overline{p_{0}}\left[\begin{array}{c}
-1 \\
1
\end{array}\right]
$$

with the coefficients:

$$
\begin{aligned}
& a_{11}=I_{0}\left(\eta_{b}\right)-I_{0}\left(\eta_{a}\right), \\
& a_{12}=K_{0}\left(\eta_{b}\right)-K_{0}\left(\eta_{a}\right), \\
& a_{21}=\hat{a} I_{0}\left(\eta_{b}\right)-\frac{\lambda_{2}}{s}\left(\eta_{b} I_{1}\left(\eta_{b}\right)-\eta_{a} I_{1}\left(\eta_{a}\right)\right), \\
& a_{22}=\hat{a} K_{0}\left(\eta_{a}\right)+\frac{\lambda_{2}}{s}\left(\eta_{b} K_{1}\left(\eta_{b}\right)-\eta_{a} K_{1}\left(\eta_{a}\right)\right),
\end{aligned}
$$

where $\eta_{b}=b \sqrt{s}, \eta_{a}=a \sqrt{s}$ and $\hat{a}=b^{2}-a^{2}$. This system is easily inverted to find the coefficients $A_{1}$ and $A_{2}$.

The Laplace transform inversion of $\bar{\phi}(r, s)$ cannot be done analytically due to the complexity of the result. However, a solution, for small times can be found by inverting the asymptotic form of $\bar{\phi}(r, s)$ as $s \rightarrow \infty$.

The asymptotic expressions for $I_{\nu}(z), K_{\nu}(z)$ as $z \rightarrow \infty$ are ([1] equations: 
9.7.1, 9.7.2):

$$
\begin{aligned}
I_{\nu}(z) & \sim \frac{e^{z}}{\sqrt{2 \pi z}}\left\{1-\frac{\mu-1}{8 z}+\frac{(\mu-1)(\mu-9)}{2(8 z)^{2}}+\cdots\right\}, \\
K_{\nu}(z) & \sim \sqrt{\frac{\pi}{2 z}} e^{-z}\left\{1+\frac{\mu-1}{8 z}+\frac{(\mu-1)(\mu-9)}{2(8 z)^{2}}+\cdots\right\},
\end{aligned}
$$

where $\mu=4 \nu^{2}$. Equation (30) is then expressed as a sum of three series

$$
\sum_{i j k} \alpha_{i j k} \frac{1}{s^{k / 2+1}} e^{( \pm r+i a-j b) \sqrt{s}},
$$

where $i, j, k$ are integers denoting the individual series and $\alpha_{i j k}$ is the appropriate coefficient. The $\pm r$ term arises from use of either $I_{0}(\eta)$ or $K_{0}(\eta)$ in equation (30). The algebraic manipulation package Maple was used to find this series. For example, the first few non-zero terms of $\bar{\phi}(r, s)$ as $s \rightarrow \infty$ are:

$$
\begin{aligned}
\bar{\phi}(r, s) \approx & e^{-(b-r) \sqrt{s}}\left\{\frac{\alpha_{010}}{s}+\frac{\alpha_{011}}{s \sqrt{s}}\right\}+e^{-(r-a) \sqrt{s}}\left\{\frac{\alpha_{100}}{s}+\frac{\alpha_{101}}{s \sqrt{s}}\right\} \\
& +e^{-(2 b-a-r) \sqrt{s}}\left\{\frac{\alpha_{120}}{s}+\frac{\alpha_{121}}{s \sqrt{s}}\right\}+e^{-(r+b-2 a) \sqrt{s}}\left\{\frac{\alpha_{210}}{s}+\frac{\alpha_{221}}{s \sqrt{s}}\right\}
\end{aligned}
$$

where each of terms $\alpha_{i j k}$ represent the appropriate coefficient calculated by Maple (but too long to list here). The solution, $\phi(r, t)$, is easily obtained using the inversion:

$$
\mathcal{L}^{-1}\left\{\frac{e^{-x \sqrt{s}}}{s^{1+k / 2}}\right\}=(4 t)^{k / 2} \mathbf{i}^{k} \operatorname{erfc}\left\{\frac{x}{2 \sqrt{t}}\right\}, \quad x>0
$$


A more complete description of the method including Maple code is given in the appendix.

\section{Steady State Solution}

The steady state solution, when the system has reached equilibrium, is found by setting $\partial \phi / \partial t=0$ in equation (23). The resulting solution is:

$$
\phi=A+B \log r,
$$

where

$$
\begin{gathered}
B=\frac{p_{0}}{\log (a / b)}, \\
A=\frac{1}{1+\lambda_{2}}\left[\frac{B\left(1-\lambda_{2}\right)}{b^{2}-a^{2}}\left(b^{2} \log b-a^{2} \log a\right)-\frac{B\left(1-\lambda_{2}\right)}{2}+\frac{2 p_{0} b^{2}}{b^{2}-a^{2}}\right] .
\end{gathered}
$$

This solution is useful in checking the numerical code and for comparing when the small time asymptotic solution, determined in the previous section, is valid.

\section{Results and Discussion}

Figure 2 illustrates the comparison of numerical with analytical techniques when $a_{0}=0.5, \lambda_{2}=0.4, b=1, k=1$ and $p_{0}=0.1$. The dilatation, $\phi$, is plotted 


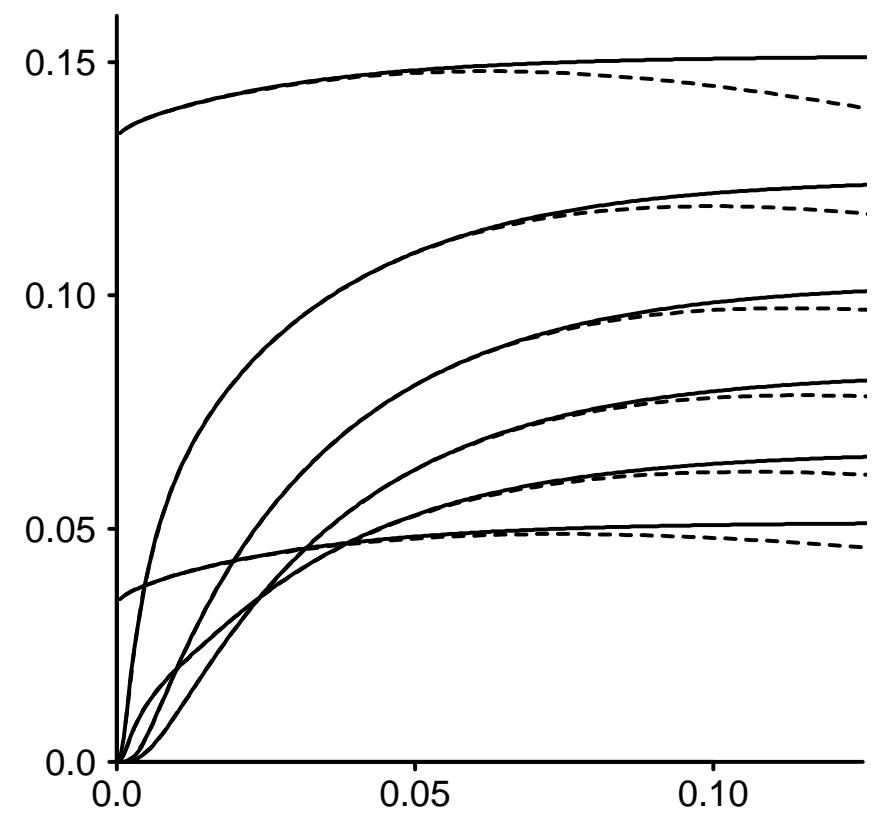

FIGURE 2. Dilatation, $\phi$, versus nondimensional time when $a_{0}=0.5, k=1.0$, $p_{0}=0.1$, and $\lambda_{2}=0.4$ for an unconstrained cylinder. The small time asymptotic solution to the linearised form of equations (23-25) are shown as dashed lines. The solid line indicated the numerical solution to the nonlinear set of equations (23-28). Note that the small time solution is in excellent agreement with the numerical solution almost until equilibrium. 
versus time for 6 different radii, $r=0.5,0.6, \ldots, 1.0$. The solid line indicates the numerical solution and the dashed line the small time asymptotic solution. The numerical method also illustrates that the steady state solution is reached at approximately $t=0.1$. There is excellent agreement between the two methods for $t<0.05$. Surprisingly, the small time asymptotic solution predicts the solution up to $t \approx 0.05$ when the material has almost reached equilibrium. Importantly, the analytical solution markedly diverges at this time, clearly illustrating the limits of the asymptotic analysis. Thus, a combination of the steady state solution with small time asymptotic solution, can give most of the information necessary in an analysis of the linearised poro-elastic equations.

The effect of the moving boundary conditions are not, however, illustrated by the linearised analysis. Figure 3 illustrates the dilatation (solid lines) found using the numerical method with a moving boundary condition (27). The parameters are $a_{0}=0.5, \lambda_{2}=0.4, k=1$ and $p_{0}=0.05$. Also shown is the comparable solution (dashed lines) using the linearised boundary conditions. Five different times are indicated for both solutions, $t=0.02,0.04, \ldots, 0.1$ where $t=0.02$ is the lower curve and $t=0.1$ is the upper curve in each set of curves. It should be noted that $\phi$ is shown in its deformed (spatial) position in each plot. For this case of the unconstrained geometry the effect of using the correct moving boundary condition is to increase the overall deformation of the cylinder and therefore to also increase the porosity. Irrespective of which boundary condition is used, the general trend in the deformation is similar. The radial deformations are larger at the inner boundary than the outer boundary. Hence, the change in porosity is larger at the inner boundary than the outer boundary. The time evolution occurs over a similar time scale for both linearised and nonlinear solution. 


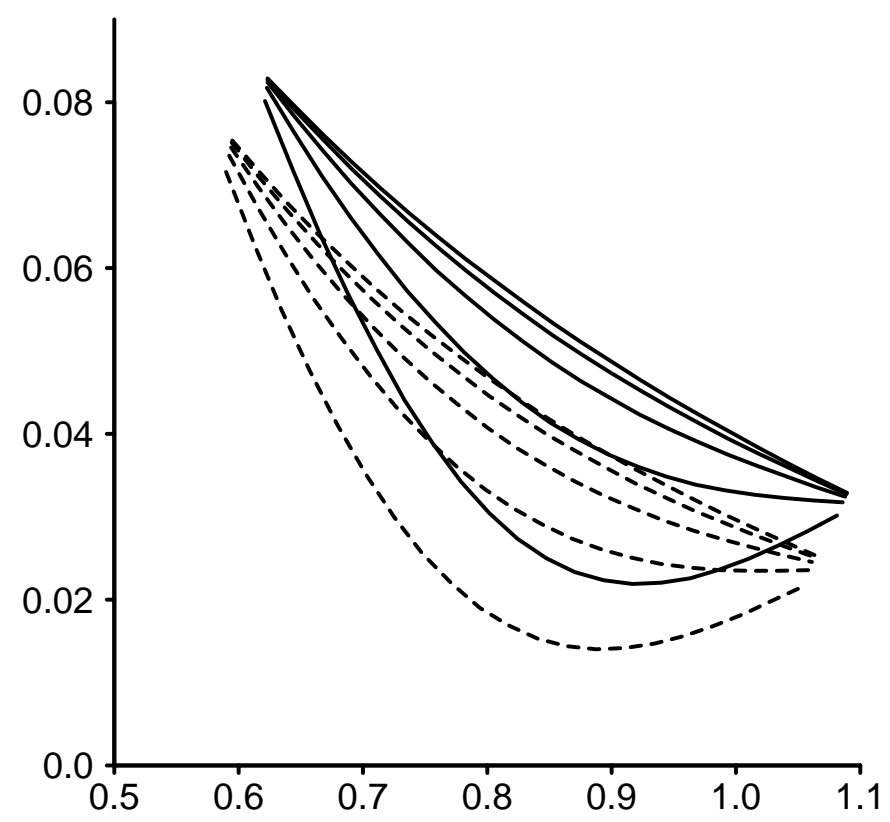

FIGURE 3. Dilatation, $\phi$, versus radial distance $r$ for an unconstrained cylinder when $a_{0}=0.5, k=1.0, p_{0}=0.05$, and $\lambda_{2}=0.4$. The solution is shown for $t=0.02,0.04, \ldots, 0.1$. The solid lines indicate the solution when the moving boundary nonlinearities are included. The dashed line represents the linearised solution. 


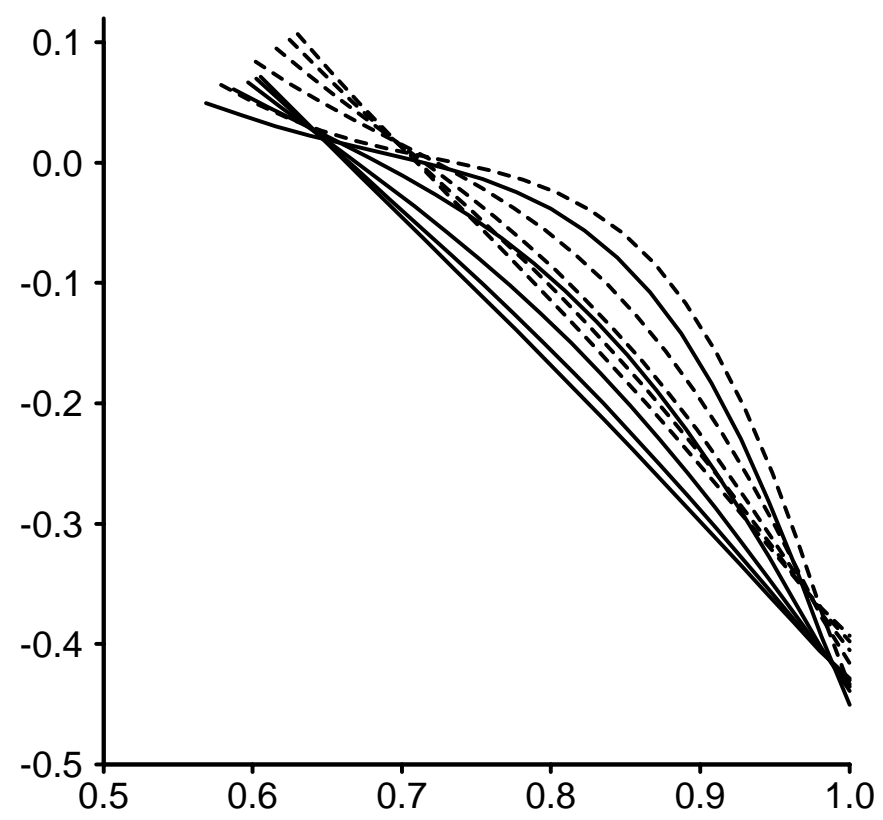

FIGURE 4. Dilatation, $\phi$, versus radial distance $r$ for a constrained cylinder when $a_{0}=0.5, k=1.0, p_{0}=0.5$, and $\lambda_{2}=0.4$. The solution is shown for $t=0.008,0.016, \ldots, 0.04$. The solid lines indicate the solution when the moving boundary nonlinearities are included. The dashed line represents the linearised solution. 
In contrast to the unconstrained geometry, the constrained geometry shown in Figure 4 , has a significant region where the medium is compressed, $\phi<0$. This figure has the same parameters as Figure 3 except $p_{0}=0.5$ and the dilatation is shown at times $t=0.008,0.16, \ldots, 0.04$ with the upper curve of each set being $t=0.008$ and the lower curve $t=0.04$. For both the linearised solution (dashed lines) and the nonlinear moving boundary solution (solid lines) there is only a small region near the inner boundary where the material is expanded. Notably, this region is smaller when the correct moving boundary is included. Note that to obtain similar deformations at the inner boundary, comparable to the unconstrained deformation in Figure 3, a substantially larger applied pressure is needed. This is highlighted in Figure 5.

We illustrate, in Figure 5, the difference in the displacement at the inner boundary, $u(a)$, at steady state, for a variety of pressures with $\lambda_{2}=0.4$ and $a_{0}=0.5$. Both the constrained and unconstrained systems are illustrated. The solid lines indicate the displacement when $k=1$ and incorporating the moving boundary. The long dash line represents the solution when $k=\exp (4 \phi)$ also with the moving boundary effect included. The fully linearised solution, without the moving boundary being considered, is shown by the short dashed line. Naturally there is a linear relationship between pressure and displacement in this latter case. Note that for the unconstrained geometry the solid and long dash lines are indistinguishable, indicating that the effect of the nonlinear permeability is negligible in comparison to the effect of the moving boundary nonlinearity. For the constrained geometry, however, the effect of the moving boundary nonlinearity is comparable to that due to the nonlinear permeability. This effect is examined in Figure 6. 


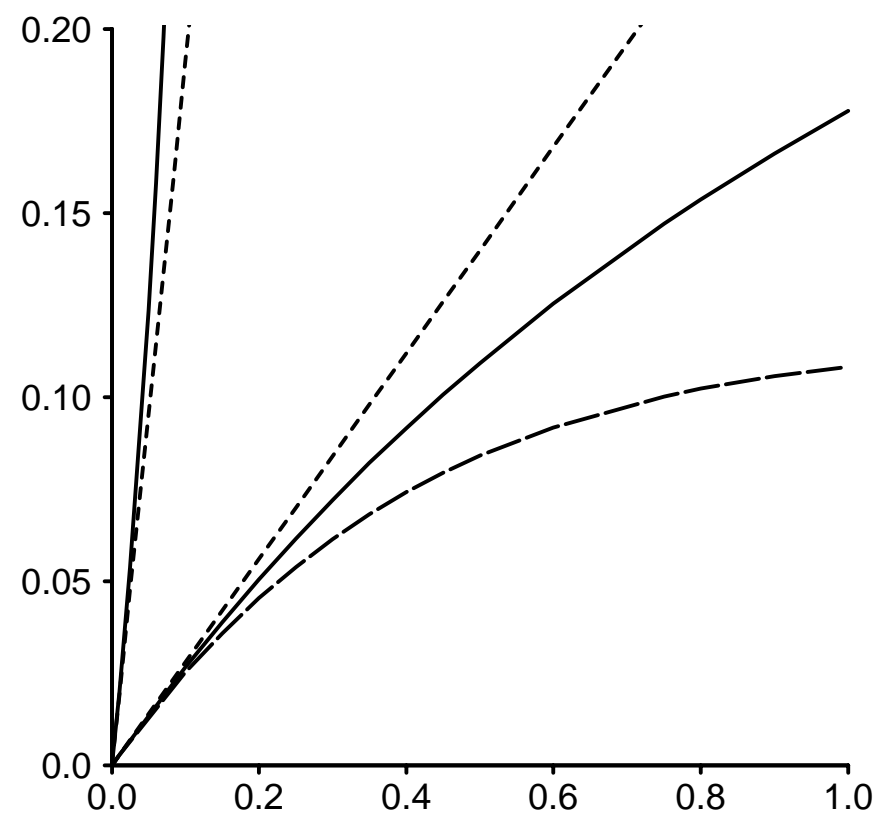

FIGURE 5. The displacement of the inner boundary at equilibrium as a function of the applied pressure when $a_{0}=0.5$ and $\lambda_{2}=0.4$. Both the constrained and unconstrained geometry are represented with the short dashed line being the linearised solution, the solid line the moving boundary solution with $k=1$, and the long dash line the solution with moving boundary solution and $k=\exp (4 \phi)$. Note that for the unconstrained cylinder the latter two solutions (solid and long dashed lines) are indistinguishable. 


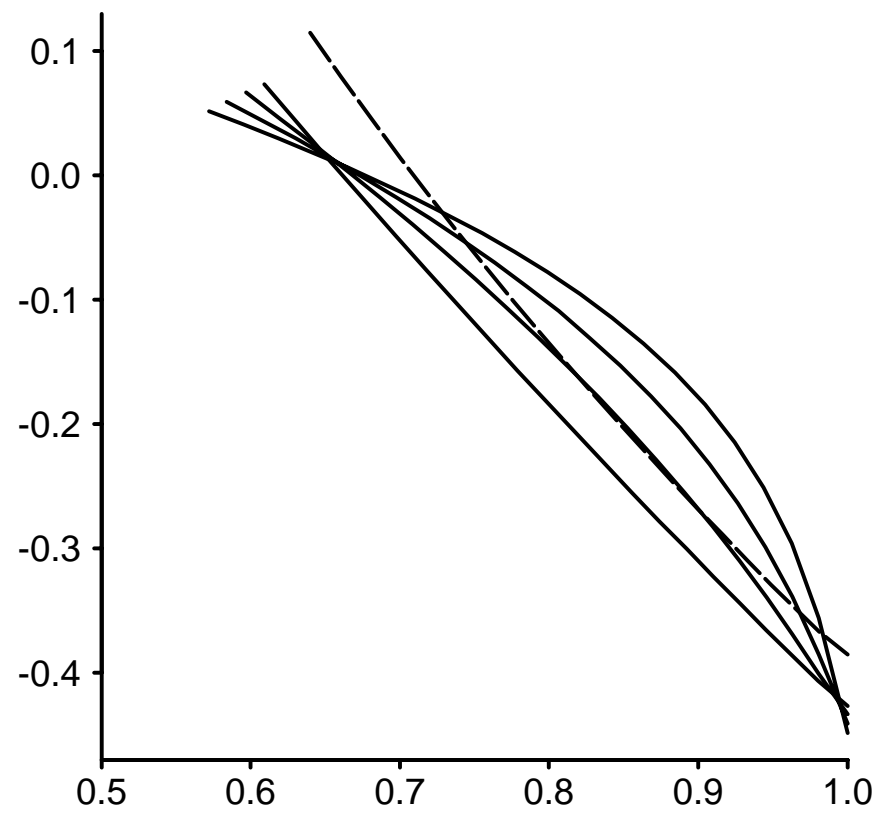

FIGURE 6. Equilibrium dilatation versus radial distance $r$ for a constrained cylinder when $a_{0}=0.5, k=\exp (m \phi), p_{0}=0.5$, and $\lambda_{2}=0.4$. The solid lines indicate solutions for (a) $m=0$, (b) $m=2$, (c) $m=4$, (d) $m=6$ when the moving boundary is considered. The dashed line indicates the fully linearised solution when $k=1$ and the boundary conditions are applied at the original position of the boundaries. 
The dilation, for the constrained geometry with moving boundary, is shown in Figure 6 with four different exponential permeabilities, $k=\exp (m \phi)$, with $m=0,2,4,6$. As a reference, the linearised solution is also shown as a dashed line. The effect of the nonlinearity clearly gives solutions that are fundamentally different to the linear problem. Note the resemblance of Figure 6 to Figure 4. It is apparent that when nonlinear permeabilities are included in the analysis, the solution progresses to a steady state that is qualitatively similar to the small time solutions of the linearised problem.

Figures 2-6 illustrate that inclusion of the boundary position, as an unknown moving boundary, has a significant effect on the dilatation and displacement. This effect is comparable in magnitude to the effect of including a nonlinear permeability. Hence any analysis of deformation in poro-elastic materials should be restricted to either a fully linearised system with very small displacements or a nonlinear analysis with both the position of the boundaries and the permeability being considered. In particular, a standard model of artery deformation with an unconstrained outer boundary, is effected more by the large movements of the boundaries than by the relatively small change in dilatation. We note, however, that we have not included any finite deformation effect due to a nonlinear stressstrain relationship. There have been a few studies of finite deformation of articular cartilage and intervertebral disks using a nonlinear stress strain relationship. These have been either simple one-dimensional studies or finite element analyses. A study of artery deformation, using realistic nonlinear stress-strain relationships and permeability, is hence desirable for a more complete understanding of the role of deformation in atherogenesis. 


\section{Conclusion}

The radial fluid flow through a porous deformable cyclinder can be modelled using poro-elasticity with the inclusion of many different nonlinearities depending on the application. Usually a nonlinear dependence of the permeability with strain is included and less often nonlinear finite deformation stress-strain relations. We have considered here the nonlinearity due to the time dependent movement of the boundaries which is not included in most poro-elastic models. We have derived the poro-elastic equations as a nonlinear diffusion equation for the dilatation with unusual integral boundary conditions and a moving boundary. These we solve numerically and show that the effect of the moving boundary is comparable to, and often larger than, the effect of including a nonlinear permeability. In particular, for an unconstrained cylinder the effect of including the nonlinear permeability is negligible compared with the effect of including the moving boundary.

Our numerical solutions show that the inclusion of a moving boundary increases the overall dilatation in an unconstrained cylinder, but decreases the dilatation when a cylinder constrained by an outer mesh is considered. In both cases the solutions including the moving boundary are qualitatively the same shape as when the moving boundary is not included despite the respective decrease and increase in dilation.

We also demonstrate how the algebraic manipulation package Maple can be used to find very accurate asymptotic solutions, for small times, for the linearised equations and that these solutions are remarkably accurate even for large times 
when the steady state solution is almost obtained.

The steady state solutions for nonlinear permeability are easily found and were shown, rather curiously, to bear a remarkable resemblance to the time dependent behaviour of the fully nonlinear moving boundary problem. Along with the qualitative similarity between solutions including and not including the moving boundary this could indicate some sort of symmetry in the governing differential equations that could be exploited in future work. A future project could be to find a simple approximation that can be used to transform the solution assuming a fixed boundary to the full moving boundary problem.

Apart from the interesting similarities between some of the solutions, our main conclusion is simple. The effect on the deformation of including the moving boundary is comparable to that of including the nonlinear permeability. Although we have studied only the cylindrical geometry, we believe that any future studies of poro-elastic behaviour that include a nonlinear permeability should also include the affect of the moving boundary.

\section{A Appendix}

We define here a methodology for using the algebraic manipulation package, Maple, to find the small time asymptotic solution to equations (23-26) with the boundary conditions applied at $a=a_{0}, b=1$ and with $k=1$. 
We first define the asymptotic expansions, equation (35), for the Bessel functions, $I_{0}, K_{0}$ :

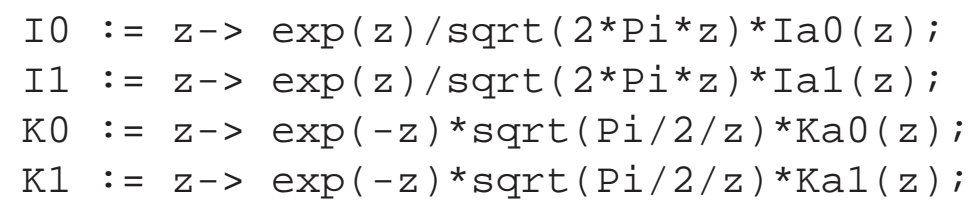

The coefficients of the matrix $\left[a_{i j}\right]$, equation (34), are then defined:

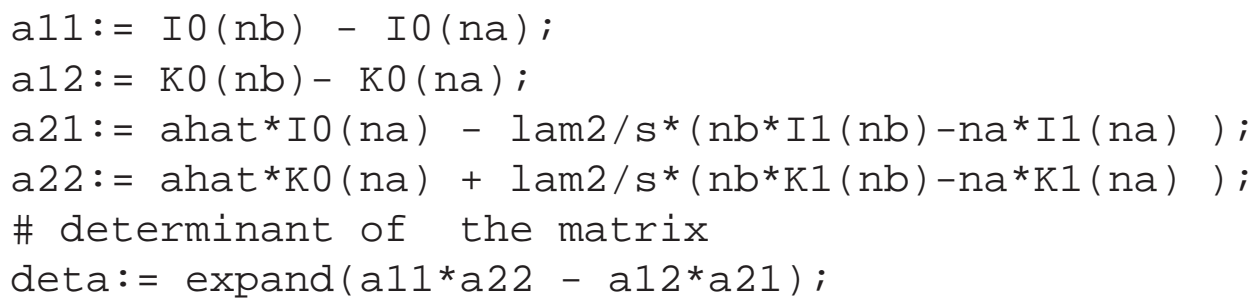

The notation 'ahat' $=\hat{a}$, 'lam2' $=\lambda_{2}$, 'nb' $=\eta_{b}=b \sqrt{s}$ and 'deta' is the determinant of the matrix $\left[a_{i j}\right]$. The determinant can be written as:

$$
\begin{aligned}
\operatorname{deta} & =c_{a b} e^{\eta_{a}-\eta_{b}}+c_{b a} e^{\eta_{b}-n a}+c_{1} \\
& =c_{b a} e^{\eta_{b}-n a}(1+\epsilon),
\end{aligned}
$$

where

$$
\epsilon=\alpha_{1} e^{-\left(\eta_{b}-\eta_{a}\right)}+\alpha_{2} e^{-2\left(\eta_{b}-\eta_{a}\right)},
$$


with

$$
\alpha_{1}=\frac{c_{1}}{c_{b a}}, \quad \alpha_{2}=\frac{c_{a b}}{c_{b a}} .
$$

To find the coefficients $c_{1}, c_{a b}$ and $c_{b a}$ we calculate:

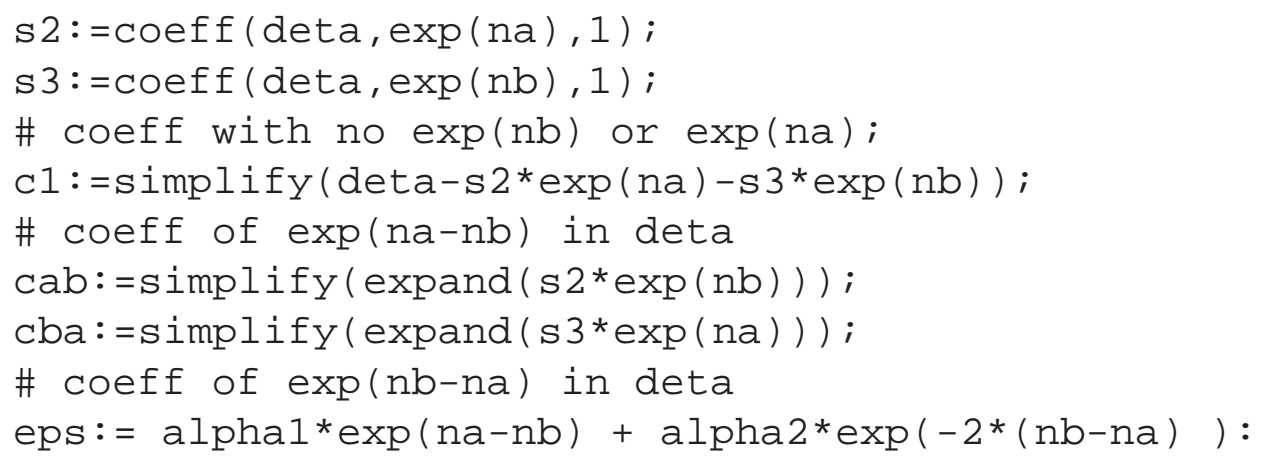

\section{Expanding}

$$
\frac{1}{1+\epsilon} \approx 1-\epsilon+\epsilon^{2},
$$

we can write:

$$
\begin{aligned}
& A_{1}=-\frac{\overline{p_{0}}}{c_{b a}} e^{-\left(\eta_{b}-\eta_{a}\right)}\left(a_{22}+a_{12}\right)\left(1-\epsilon+\epsilon^{2}\right), \\
& A_{2}=\frac{\overline{p_{0}}}{c_{b a}} e^{-\left(\eta_{b}-\eta_{a}\right)}\left(a_{21}+a_{11}\right)\left(1-\epsilon+\epsilon^{2}\right) .
\end{aligned}
$$

We now establish a series of powers of $e^{\eta_{a}}$ and $e^{-\eta_{b}}$. 
$\mathrm{n}:=2$ : \# the highest term in the series of

\# exponentials exp(ia-jb).

$\mathrm{m}:=1$ : \# the highest term in the series $1 / \mathrm{s}^{\wedge}(\mathrm{k} / 2)$.

\# part of A_1

top $1:=\operatorname{expand}\left(\exp (n a-n b) *(a 22+a 12) *\left(1-e p s+e p s^{\wedge} 2\right)\right)$;

\# part of A_2

top2: = expand $\left(\exp (n a-n b) *(a 21+a 11) *\left(1-e p s+e p s^{\wedge} 2\right)\right)$;

\# defining new variables for

\# evaluating a series expansion.

$\mathrm{h} 1:=\operatorname{subs}(\{\exp (\mathrm{na})=\mathrm{xx}, \exp (\mathrm{nb})=1 / \mathrm{yy}\}$, top 1$)$;

$\mathrm{h} 2:=\operatorname{subs}(\{\exp (\mathrm{na})=\mathrm{xx}, \exp (\mathrm{nb})=1 / \mathrm{yy}\}$, top 2$) ;$

for $i$ from 0 to $n$ do

for $j$ from 0 to $n$ do

\# coeff of $\exp \left(i{ }^{*} n a-j{ }^{*} n b\right)$ in top 1

$a[i, j]:=\operatorname{coeff}(\operatorname{coeff}(h 1, x x, i), y y, j) ;$

\# coeff of exp (i*na-j*nb) in top2

$b[i, j]:=\operatorname{coeff}(\operatorname{coeff}(h 2, x x, i), y y, j) ;$

od; od;

For each of these terms $e^{i \eta_{a}-j \eta_{b}}$ we must find the coefficient as a series in terms of powers of $\frac{1}{\sqrt{s}}=$ 'oss'. We now define a few terms:

alpha1 $:=c 1 / c b a:$ alpha2 $:=c a b / c b a:$

$\mathrm{na}:=\mathrm{a} /$ oss; $\mathrm{nb}:=\mathrm{b} /$ oss; $\mathrm{nr}:=\mathrm{r} / \mathrm{oss} ;$

$s:=1 /\left(\mathrm{oss}^{\wedge} 2\right)$; 
\# The series expansions of the Bessel functions.

\begin{tabular}{|c|c|c|}
\hline$:=$ & $->$ & \\
\hline & & $z /(8 * z)^{\wedge} 2-5 *$ \\
\hline & & z) $2-3 *$ \\
\hline
\end{tabular}

The solution, equation (30), is separated into two components $A_{1} I_{0}\left(\eta_{r}\right) e^{-\eta_{r}}$ and $A_{2} K_{0}\left(\eta_{r}\right) e^{\eta_{r}}$ using the asymptotic expansions (35). This can be inverted since the solution is expressed as:

$$
\bar{\phi}(r, s)=\sum_{i j k}\left(e^{r+i a-j b} \frac{1}{s^{1+k / 2}} B_{1}(i, j, k)+e^{-r+i a-j b} \frac{1}{s^{1+k / 2}} B_{2}(i, j, k)\right)
$$

where most of the coefficients $B_{1}, B_{2}$ are zero.

$\mathrm{F}:=(\mathrm{n}, \mathrm{x})->(4 * t)^{\wedge}(\mathrm{n} / 2) \star \operatorname{erfc}(\mathrm{n},-\mathrm{x} / 2 / \operatorname{sqrt}(\mathrm{t})) ;$

soln $:=0:$

for $i$ from 0 to $n$ do

for $j$ from 0 to $n$ do

$\operatorname{cc} 1[i, j]:=\operatorname{series}(-a[i, j] / c b a / \operatorname{sqrt}(2 * P i \star n r)$

$\left.{ }^{\star} \mathrm{IaO}(\mathrm{nr}), \mathrm{OSS}\right)$;

$\operatorname{cc} 2[i, j]:=\operatorname{series}(b[i, j] / c b a * \operatorname{sqrt}(P i / 2 / n r)$

${ }^{\star} \mathrm{KaO}(\mathrm{nr})$, oss);

for $\mathrm{k}$ from 0 to $\mathrm{m}$ do

B1 $[i, j, k]:=\operatorname{coeff}(\operatorname{cc} 1[i, j]$, oss,$k)$; 


$$
\begin{aligned}
& \text { B2 }[i, j, k]:=\operatorname{coeff}(\operatorname{cc} 2[i, j], \text { oss }, k) ; \\
& \text { soln:= soln }+ \text { po*B1 }[i, j, k] * F(k, r+i * a-j * b)+ \\
& \text { po*B2 }[i, j, k] * F(k,-r+i * a-j * b) ;
\end{aligned}
$$

od; od; od;

\# defining the solution phi $(r, t)$

phi:=unapply $(\operatorname{soln}, r, t)$;

The steady state solution is also easily evaluated using Maple.

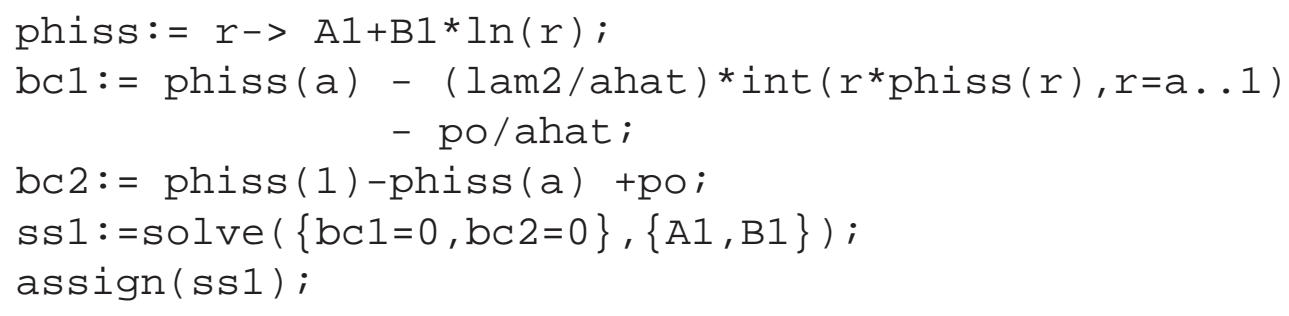

The solutions can then be plotted when the remaining parameters are defined:

$\mathrm{a}:=0.5: \mathrm{b}:=1:$ ahat $:=1-\mathrm{a}^{\wedge} 2: \operatorname{lam} 2:=0.4:$

po:=0.1: \# the scaled pressure.

plot (\{phi (r,0.0125), phi (r,0.0375), phi (r,0.0625),

phiss (r)\}, $r=a . . b)$;

\# plotting the solultion at times

\# $t=0.0125,0.0375,0.0625$

\# and at steady state. 


\section{References}

[1] M. Abramowitz and I. A. Stegun, Handbook of Mathematical Functions. (Dover, New York, 1972.)

[2] S. I. Barry and G. K. Aldis, "Unsteady flow induced deformation of porous materials.” Int. J. Non-Linear Mech. 26, (1991) 687-699.

[3] S. I. Barry and G. K. Aldis, "Comparison of models for flow induced deformation of soft biological tissue." J. Biomechanics 23, (1990) 647-654.

[4] S. I. Barry and G. K. Aldis, "Radial flow through deformable porous shells." J. Austr. Math. Soc. B 34, (1993) 333-354.

[5] M. A. Biot, "General theory of three-dimensional consolidation." J. appl. Phys. 12, (1941) 155-164.

[6] J. S. Hou, M. H. Holmes, W. M. Lai and V. C. Mow, "Boundary conditions at the cartilage-synovial fluid interface for joint lubrication and theoretical verifications." J. Biomech. Engng. 111, (1989) 78-87.

[7] M. H. Holmes, "A nonlinear diffusion equation arising in the study of soft tissue." Quart. Appl. Math. 61, (1983) 209-220.

[8] M. H. Holmes, "A theoretical analysis for determining the nonlinear hydraulic permeability of a soft tissue from a permeation experiment." Bull. Math. Biology 47, (1985) 669-683. 
[9] M. H. Holmes, "Finite deformation of soft tissue: Analysis of a mixture model in uni-axial compression." J. Biomech. Engng. 108, (1986) 372-381.

[10] R. Jain and G. Jayaraman, "A theoretical model for water flux through the arterial wall.” J. Biomech. Engng. 109, (1987) 311-317.

[11] G. Jayaraman, "Water transport in the arterial wall-a theoretical study." $J$. Biomechanics 16, (1983) 833-840.

[12] O. E. Jensen, M. R. Glucksberg, J. R. Sachs and J. B. Grotberg, "Weakly nonlinear deformation of a thin poroelastic layer with a free surface." J. Appl. Mech. 61, (1994) 729-730.

[13] D. E. Kenyon, "The theory of an incompressible solid-fluid mixture." Arch. Rat. Mech. Anal. 62, (1976) 131-147.

[14] D. E. Kenyon, "Transient filtration in a porous elastic cylinder." J. Appl. Mech. 98, (1976) 594-598.

[15] D. E. Kenyon, "A mathematical model of water flux through aortic tissue." Bull. Math. Biology 41, (1979) 79-90.

[16] M. Klanchar and J. M. Tarbell, "Modeling water flow through arterial tissue.” Bull. Math. Biology 49, (1987) 651-669.

[17] W-S. Kim and J. M. Tarbell, "Macromolecular transport through the deformable porous media of an artery wall." J. Biomech. Engng. 116, (1994) 156-163. 
[18] W. M. Lai and V. C. Mow, "Drag induced compression of articular cartilage during a permeation experiment." Biorheology 17, (1980) 111-123.

[19] W. M. Lai, V. C. Mow and V. Roth, "Effects of nonlinear strain-dependent permeability and rate of compression on the stress behaviour of articular $631,631,631$ cartilage." J. Biomech. Engng. 103, (1981) 61-66.

[20] M. J. Lever and M. T. Jay, "Convective and diffusive transport of plasma proteins across the walls of large blood vessels." Frontiers Med. Biol. Engng. 5,(1993) 45-50.

[21] A. F. Mak, “The apparent viscoelastic behaviour of articular cartilage-The contributions from the intrinsic matrix viscoelasticity and interstitial fluid flows." J. Biomech. Engng. 108, (1986) 123-130.

[22] V. C. Mow, M. H. Holmes and W. M. Lai, "Fluid transport and mechanical properties of articular cartilage: a review." J. Biomechanics 17, (1984) 377631 394.

[23] V. C. Mow, M. K. Kwan, W. M. Lai and M. H. Holmes, "A finite deformation theory for nonlinearly permeable soft hydrated biological tissues." Frontiers in Biomechanics (eds. G. Schmid-Schoenbein, S. L-Y. Woo and B. W. Zweifach), (Springer-Verlag, New York, 1985), 153-179.

[24] R. M. Nerem and J. F. Cornhill, "The role of fluid mechanics in artherogenesis". J. Biomech. Engng. 102, (1980) 181-189. 
[25] G. M. Saidel, E. D. Morris and G. M. Chisolm III, "Transport of macromolecules in arterial wall in vivo: A mathematical model and analytical solutions." Bull. Math. Biol. 49, (1987) 153-169.

[26] L. A. Taber, "Axisymmetric deformation of poroelastic shells of revolution." Int. J. Solids Structures 29, (1992) 3125-3143.

[27] K. Terzaghi, Erdbaumechanik auf Bodenphysikalischen Grundlagen. (Wien, Deuticke, 1925). 